\title{
Quantitative Z-contrast Imaging in Scanning Transmission Electron Microscopy of Zeolite-supported Metal Clusters and Single-metal-atom Complexes With Single-Atom Sensitivity
}

\author{
Pinghong $\mathrm{Xu}^{1}$, Claudia Martinez-Macias ${ }^{1}$, Joey Kistler ${ }^{1}$, Nutchapon Chotigkrai ${ }^{2}$, Bruce C. Gates ${ }^{1}$, Nigel \\ D. Browning ${ }^{3}$ \\ ${ }^{1}$ Department of Chemical Engineering and Materials Science, University of California, Davis, Davis, \\ CA 95616, USA \\ ${ }^{2}$ Department of Chemical Engineering, Chulalongkorn University, Bangkok 10330, Thailand \\ ${ }^{3}$ Fundamental and Computational Science Directorate, Pacific Northwest National Laboratory, \\ Richland, WA 99352, USA
}

Supported metal catalysts, in particular noble metals supported on zeolites and oxides, are widely applied in large-scale processes, such as petroleum refining, petrochemical conversion, and automobile exhaust conversion. A crucial challenge in studying these catalysts lies in their complex nature, including non-uniform supports, variable sizes, shapes, and distributions of metal species and multiplicities of ligands. Therefore, it is essential to investigate supported metal catalysts with small, uniform structures on highly crystalline supports to gain fundamental understanding of supported catalysts [1-2]. In this work, zeolites were chosen as the support material because of their wide applications in industry and their high degrees of crystallinity, which provide well-defined structures for imaging. High-angle annular dark field scanning transmission electron microscopy (HAADF-STEM) is well-suited to characterizing this type of material because the high atomic number difference between the support and the supported metal provides a strong contrast in the STEM images [3]. Here we present direct measurements of such catalysts, include metal size and distribution within the support, using aberration-corrected STEM at atomic resolution with single atom sensitivity. Quantitative analysis of metal sizes, shapes and their bonding locations within the cavities of zeolite structures was performed, which demonstrated the advantages of the STEM technique. Extended X-ray absorption fine structure and infrared spectroscopies were also applied to obtain complementary information, such as identification of ligands, determination of metal-metal distances and coordination number.

Currently there are two main approaches applied for microscopic investigations of zeolite-supported metal catalysts - the use of low dose EM techniques or high dose EM techniques. High electron-dose $\left(10^{5}-10^{8} \mathrm{e}^{-} / \mathrm{A}^{2}\right)$ STEM imaging of these highly beam sensitive materials provides high signal-to-noise ratio for easy interpretation, whereas low dose STEM imaging usually requires intense image processing [4]. Here we demonstrate the advantages of high-dose STEM imaging, with images taken quickly before significant destruction or modification of of structures. Results of two samples will be presented, namely (1) dealuminated HY zeolite-supported mononuclear iridium species with CO ligands (Figure 1) and (2) zeolite KLTL-supported platinum complexes with amine ligands (Figure 2). In both cases, the interactions between the zeolite supports and the metal species was revealed in detail. Moreover, the influence of the electron beam on the samples was carefully examined, to provide information about the metal-support bonding and the metal species of various sizes at different crystallographic locations in the sample [5]. 


\section{References:}

[1] N. D. Browning at al, ChemCatChem 2013, 5, 2673-2683

[2] C. Aydin at al, J. Phys. Chem. Lett. 2013, 3, 1865-1871

[3] A. A. Herzing et al, Science 2008, 321, 1331

[4] V. Ortalan et al, Nature Nanotechnology, 2010, 5, 506

[5] This work is supported by the U.S. Department of Energy Grant No. DE-FG02-03ER46057 and Grant No. DE-FG0204ER15513 (C.M.M). C.M.M. was partially supported by the UC

MEXUS-CONACYT doctoral fellowship program. The research described in this paper is part of the Chemical Imaging Initiative at PNNL under Contract DE-AC05-76RL01830 operated for DOE by Battelle. It was conducted under the Laboratory Directed Research and Development Program at PNNL, a multiprogram national laboratory operated by Battelle for the U.S. Department of Energy. A portion of the research was performed using EMSL, a national scientific user facility sponsored by the Department of Energy's Office of Biological and Environmental Research and located at Pacific Northwest National Laboratory.

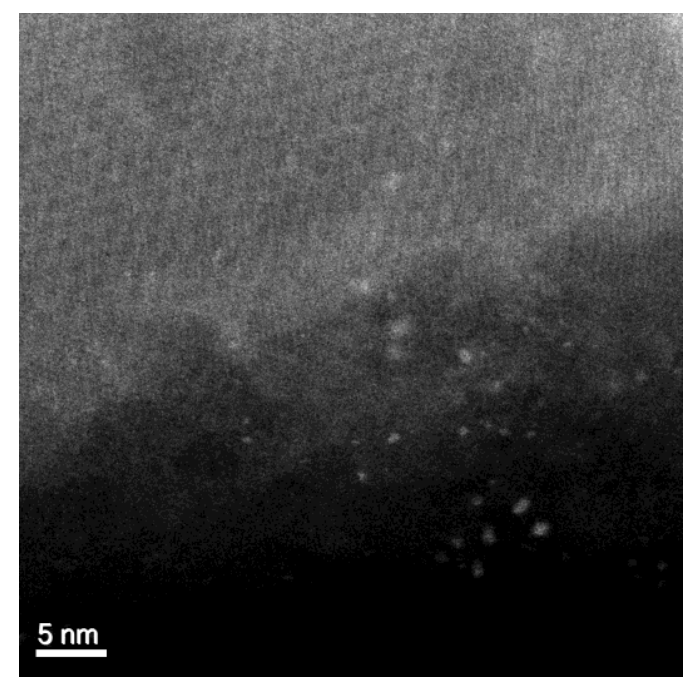

Figure 1. STEM image characterizing $\operatorname{Ir}(\mathrm{CO})_{2} / \mathrm{HY}$ supported catalyst.

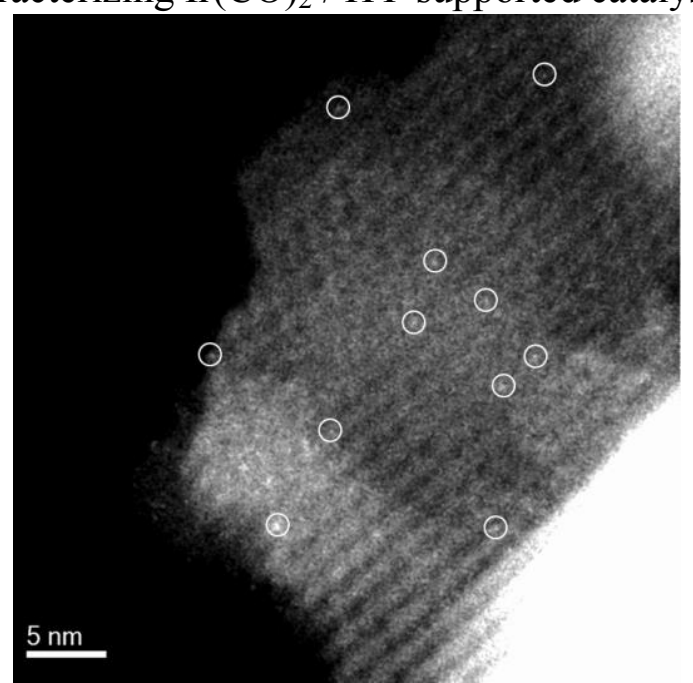

Figure 2. STEM image characterizing $\mathrm{Pt}\left(\mathrm{NH}_{3}\right)_{4}$ / LTL supported catalyst. Pt mononuclear species are encircled. 